\title{
Rechtserhaltende Gewalt oder rechtserhaltender Zwang - mehr als eine semantische Unterscheidung?
}

Mit der Konzeptualisierung der rechtserhaltenden Gewalt in Abgrenzung zum gerechten Krieg (,,Rechtserhaltende Gewalt“ statt ,gerechter Krieg ““, EKD 2007, Ziff. 65) liegt der Fokus der friedensethischen Debatten - und das hat das vorherige Kapitel deutlich aufzeigen können - auf Fragen legaler und legitimer Gewalt und damit auf militärische Interventionen. Dieser Fokus ist nachvollziehbar und stellt auch einen zentralen Aspekt in friedensethischen Debatten dar, ,verstellt aber den Blick auf Formen der politischen Einflussnahme jenseits militärischer Gewalt, die nichtsdestotrotz normativ rechtfertigungsbedürftig sind [...]: Formen des politischen Zwangs“ (Daase 2016, S. 12; vgl. auch Daase 2019, S. 23).

Der Terminus des Zwangs ist kein originär friedenswissenschaftlicher, der Begriff findet sich verstärkt in Rechtsdiskursen. Im Rahmen des gerechten Friedens mit seinem explizit rechtsethischen Zugang (,Frieden durch Recht“) liegt seine Verwendung aber auch nicht fern. Mit ihm verbindet sich vor allem die Chance, infolge des veränderten Fokus und der Perspektiverweiterung über bisherige friedenspolitische und -ethische Debatten hinauszugelangen. So gehe es ,in vielen Institutionen und auch im internationalen Recht [...] nicht in erster Linie um die Durchsetzung von Normen mit Gewalt, sondern um die Stärkung ihrer Verbindlichkeit durch Androhung von Sanktionen, also um Zwangsbewährung“ (Daase 2016, S. 12).

Allgemein bezeichnet Zwang ,die Stärke, jemanden gefügig zu machen“ (Imbusch 2002, S. 33). Der durch „die Androhung physischer Eingriffe bzw. bestimmter Erzwingungsmittel“ (Imbusch 2002, S. 33) erzeugte Druck soll jemanden dazu veranlassen, entweder Fremdbestimmtes tun zu müssen (gebietender Zwang) oder Selbstbestimmtes nicht tun zu dürfen (verbietender Zwang) (vgl. Batthyány 2007, S. 153). Dabei stellt Gewalt in seinem Verständnis als direkte physische Gewalt nur „eine bestimmte Form des Zwanges neben anderen“ und „ein spezifisches Mittel der Nötigung unter übrigen“ (Neidhardt 1986, S. 132) dar. 
In der internationalen Politik kommt eine ganze Bandbreite von Maßnahmen in Betracht; angefangen vom psychischen Druck und dem Beschämen (shaming and blaming) über die Androhung oder Durchführung nicht-gewaltsamer Sanktionen wie Wirtschaftsboykotte oder den Ausschluss aus Gremien und Organisationen bis hin zu militärischen Interventionen als ultima ratio (vgl. Daase 2019, S. 24).

Insbesondere die Sanktionspolitik als ein „Instrument des internationalen Krisen- und Konfliktmanagements" (Werthes 2019, S. 122) bedarf - eingehender als bisher geschehen - der friedensethischen Beachtung und Reflexion. Denn obwohl Sanktionen in der internationalen Politik und Praxis zunehmen, ist es bislang noch nicht gelungen, einen formalen, das heißt rechtlich geregelten und vorhersagbaren Sanktionsmechanismus zu etablieren (vgl. Werthes 2019, S. 129 f.). Dabei können die Folgen verhängter Sanktionen für die betroffenen Staaten und ihre Gesellschaften durchaus gravierend sein; gegebenenfalls kommen sie gar einem „genocidal tool“" (Simons 1999, S. xi; vgl. auch Werthes 2019, S. 132) gleich.

Auch Sanktionen (lat. sancire, übersetzt mit heiligen, festsetzen, bestätigen) sind vielfältig; sie können als „Reaktionen anderer auf normgemäßes oder von der Norm abweichendes Verhalten eines Sanktionsadressaten“ (Werthes 2019, S. 122 f.) gefasst werden. Im Fokus steht die „Rechtfertigung, Begründung und Bestätigung von Normen“ (Daase 2016, S. 12). Das kann in zweierlei Weise erfolgen: bei Verstoß des Adressaten durch Missbilligung und Strafe oder aber im Sinne der Bestätigung einer Norm durch Anreiz und Belohnung. Sanktionen haben zwei zentrale Funktionen inne: Zum einen ist es die ,willensbeugende Funktion" (Werthes 2019, S. 125). Mit ihr verbindet sich die Erzwingung eines bestimmten Verhaltens, „um eine allgemeine Norm zur Geltung zu verhelfen“ (Daase 2016, S. 13). Aber auch „machtlose Sanktionen sind Sanktionen“ (Daase 2016, S. 13, 2019 S. 28). So kommt ihnen - zweitens - eine normative Funktion zu. Hier gilt es, ,,den sanktionierten Akteur zu stigmatisieren und ihm , die Missbilligung seines Verhaltens durch die Staatengemeinschaft deutlich vor Augen zu führen ' (Kulessa und Starck 1997, S. 4)“(Werthes 2019, S. 125). In diesem Sinne verstehen sich Sanktionen als ein „Element internationaler (normativer) Kommunikation“ (Werthes 2019, S. 128) mit dem Ziel, die Gültigkeit einer Norm zu bekräftigen (vgl. Daase 2016, S. 13, 2019 S. 28). Ausgehend von diesen beiden Hauptfunktionen lassen sich idealtypisch vier theoretische Wirkungsmodelle von Sanktionen unterscheiden (vgl. Werthes 2019, S. 126 ff.):

- Das klassische Modell geht von der Annahme aus, dass der Effekt von Sanktionen proportional mit den durch Sanktionen verursachten (wirtschaftlichen) Schäden zunehme, da rational agierende Regierungen auf Druck reagieren und 
ihre Politik entsprechend anpassen. Übersehen werde dabei, dass infolge der Unterdrückungsapparate autoritärer Staaten Sanktionen vorrangig zulasten der Bevölkerung gehen.

- Das interessenpluralistische Modell folgt derselben Logik, versucht aber, mittels zielgerichteter Sanktionen nicht die Bevölkerung, sondern die politisch Verantwortlichen zu treffen. Auch hier werde unterschätzt, dass ,staatliche Akteure mittels Anpassungs- und Verteilungsmaßnahmen durchaus die Effekte von gezielten Sanktionen konterkarieren oder zumindest minimieren können“ (Werthes 2019, S. 127).

- Das Modell der Ressourcenverweigerung basiert auf einer anderen Wirkungslogik. Hier werde versucht, den politisch Verantwortlichen die materiellen Ressourcen zur Fortführung ihrer Politik vorzuenthalten. Als klassisches Beispiel gelten Waffenembargos. Und auch hier gilt, dass ,die Verfolgung der sanktionierten Politik von der Versorgung mit diesen materiellen Ressourcen abhängig ist" (Werthes 2019, S. 128).

- Und das vierte Modell, das auf der normativen Funktion von Sanktionen beruht, muss sich ebenfalls der Kritik stellen, wenn „ein solcher kommunikativer Akt über symbolische Sanktionsmaßnahmen hinausgeht und Unschuldige leiden müssen“ (Werthes 2019, S. 128).

Mit Sanktionen gehen - das haben die verschiedenen Wirkungsmodelle deutlich machen können - zentrale ethische Fragen über die Angemessenheit der Reaktion einher. Umstritten ist, inwieweit diese sich nach den Kriterien rechtserhaltender Gewalt beurteilen lassen. Während Sascha Werthes (2019) diesen Versuch unternimmt, sprechen sich andere Autorinnen und Autoren mit aller Vehemenz dagegen aus. Nach Elizabeth Ellis (2020) gebe es „crucial differences between war and economic sanctions“. Dabei seien die Kriterien des gerechten Krieges und diese sind identisch mit denen der rechtserhaltenden Gewalt - ,inappropriate for use in the moral assessment of economic sanctions". Nötig für die ethischen Abwägungen der Mittel und ihrer Konsequenzen sei ein angemessenes ethisches Bezugssystem. In diesem Sinne fordert auch Christopher Daase (2019, S. 29) „eine neue Ethik des Sanktionierens als legitime Zwangspolitik“ und konstatiert:

„Im rechtsethischen Diskurs der letzten Jahre ist zu viel über den gerechten Krieg und zu wenig über legitimen Zwang gesprochen worden. Dadurch sind die Möglichkeiten konzeptionell eingeschränkt worden, auf Rechtsbrüche, Menschenrechtsverletzungen und Ungerechtigkeiten in der internationalen Politik zu reagieren.“ 
Open Access Dieses Kapitel wird unter der Creative Commons Namensnennung 4.0 International Lizenz (http://creativecommons.org/licenses/by/4.0/deed.de) veröffentlicht, welche die Nutzung, Vervielfältigung, Bearbeitung, Verbreitung und Wiedergabe in jeglichem Medium und Format erlaubt, sofern Sie den/die ursprünglichen Autor(en) und die Quelle ordnungsgemäß nennen, einen Link zur Creative Commons Lizenz beifügen und angeben, ob Änderungen vorgenommen wurden.

Die in diesem Kapitel enthaltenen Bilder und sonstiges Drittmaterial unterliegen ebenfalls der genannten Creative Commons Lizenz, sofern sich aus der Abbildungslegende nichts anderes ergibt. Sofern das betreffende Material nicht unter der genannten Creative Commons Lizenz steht und die betreffende Handlung nicht nach gesetzlichen Vorschriften erlaubt ist, ist für die oben aufgeführten Weiterverwendungen des Materials die Einwilligung des jeweiligen Rechteinhabers einzuholen.

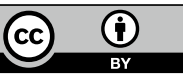

